\title{
Uso indiscriminado dos opioides e suas consequências
}

\author{
Indiscriminate use of opioids and their consequences
}

\author{
Lorena Santos Sousa* ${ }^{\circ}$, Milena Silva Cerqueira Pinheiro ${ }^{\circ}$, Juliana Lima Gomes Rodrigues ${ }^{\circ}$ \\ Universidade Salvador, Salvador, Bahia, Brasil. *Autor para correspondência. E-mail: loresousa98@gmail.com
}

\begin{abstract}
Resumo: Introdução: Os opioides pertencem a uma classe de fármacos utilizados como analgésicos para dores agudas e crônicas, são classificados em naturais, semissintéticos e sintéticos. São prescritos no tratamento da dor pós-operatória e crônica quando não há resposta de analgésicos comuns ou mais fracos, sendo eficazes nos manejos dolorosos, contudo o uso de forma indiscriminada traz diversos riscos à saúde tanto a nível físico como psicológico. Revisão: A partir da metodologia foram selecionados 34 artigos por estarem de acordo com o tema. Trata-se de uma revisão de literatura sistemática, a busca foi feita através das bases de dados Google Acadêmico e Scientific Eletronic Library Online. Discussão: São drogas bastante administradas por sua potente e rápida ação, contudo os efeitos adversos dos opioides também são comuns, entre eles: dependência, depressão respiratória, constipação intestinal, prurido, sonolência e náuseas/vômitos. Prescrever opioides de forma consciente e racional, ainda é um desafio para os profissionais de saúde capacitados. Considerações finais: Os farmacêuticos podem contribuir para prescrições adequadas de opioides, entendendo também as necessidades do acesso aos opioides, portanto, são imprescindíveis mais pesquisas sobre dor e o distúrbio do uso abusivo dessas drogas, reforço constante na educação de prescritores e garantia de acesso aos tratamentos eficazes desses medicamentos.
\end{abstract}

Palavras-chave: efeitos toxicológicos, opioides, uso indiscriminado.

\begin{abstract}
Introduction: Opioids belong to a class of drugs used as analgesics for acute and chronic pain, are classified as natural, semi-synthetic and synthetic. They are prescribed in the treatment of postoperative pain and chronic when there is no response of common or weaker analgesics being effective in painful managements, however the use indiscriminately rings several health risks both physical and psychological. Review: From the methodology, 34 articles were selected because they were in accordance with the theme. This is a systematic literature review the search was done through the Google Academic and Scientific Electronic Library Online databases. Discussion: They are drugs widely administered for their powerful and rapid action, however, the adverse effects of opioids are also common, among them: respiratory depression, constipation, itching, drowsiness and nausea/vomiting. Prescribing opioids consciously and rationally is still a challenge for trained health professionals. Final considerations: Pharmacists may contribute to adequate prescriptions of opioids also understanding the needs of access to opioids so more research on pain and the disorder of the abuse of these drugs is essential, constant reinforcement in the education of prescribers and guarantee access to effective treatments of these medicines.
\end{abstract}

Keywords: toxicological effects, opioids, indiscriminate use.

\section{Introdução}

Os opioides pertencem a uma classe de fármacos utilizados como analgésicos para dores agudas e crônicas, classificados em naturais, semissintéticos e sintéticos (Neves, 2016). Essas substâncias têm estruturas químicas diferentes, porém ações semelhantes, ao reagirem com os receptores específicos. Atuam no sistema nervoso central (cérebro e medula espinhal), se assemelhando às funções intrínsecas do próprio organismo (Bicca et al., 2012).

Produzem analgesia atuando em três receptores: MOP (receptor peptídico opioide mu), KOP (receptor peptídico opioide kappa) e DOP (receptor peptídico opioide delta), sendo estes acoplados à proteína G inibitória. Todos esses receptores estão associados ao sistema dopaminérgico e também podem ser encontrados no trato gastrointestinal (TGI) (Wiffen et al., 2017; Bezerra et al., 2018).

São prescritos no tratamento da dor pós-operatória e crônica quando não há resposta de analgésicos comuns ou mais fracos, podendo-se utilizar a morfina, além de fentanil, tramadol, codeína e metadona (Kraychete et al., 2014). Outros fármacos desta classe são: hidromorfona, oxicodona e meperidina. Segundo 
Minson et al. (2012), às doses adequadas são aquelas que trazem melhora da dor e possuem menos efeitos colaterais ao serem administradas.

São muitos os efeitos toxicológicos que a utilização exacerbada desses fármacos pode causar. Um deles é a dependência física e/ou psicológica, que consiste na necessidade da substância psicoativa provocada pela adaptação do organismo ao fármaco. A dependência pode levar à síndrome de abstinência, que é caracterizada pelo aparecimento de sintomas, quando há uma interrupção abrupta do medicamento. Já a tolerância é quando há a necessidade de doses cada vez mais crescentes para se obter os mesmos efeitos iniciais, ou diminuição dos efeitos desejados com a mesma dose (Nascimento \& Sakata, 2011). Contudo, os impactos desses efeitos precisam de maiores discussões, que levem a orientações seguras, sobre as ameaças que a utilização por longos períodos pode levar a saúde (Melo et al., 2020a). Segundo Silva et al. (2020a) o uso indiscriminado geralmente é decorrente da automedicação, além de prescrições incorretas.

$\mathrm{O}$ uso indiscriminado traz diversos riscos à saúde tanto a nível físico como psicológico, onde muitas vezes os pacientes saem de uma terapêutica para outra, visando tratar a dependência e até mesmo abstinência que essas drogas causam. Com isso, o presente trabalho tem como objetivo realizar revisão de literatura acerca dos efeitos relacionados ao uso indiscriminado de medicamentos opioides, a fim de discutir uma melhor forma de adaptação e uso desses medicamentos, avaliando a importância do profissional farmacêutico neste âmbito.

\section{Revisão}

Este trabalho trata-se de uma revisão de literatura. Para composição deste estudo foram utilizados artigos para obtenção de informações sobre o tema proposto, utilizando a palavra-chave "opioides" combinada com "uso indiscriminado", "efeitos toxicológicos e farmacológicos". A coleta se deu principalmente através da plataforma Google Acadêmico. Em relação à escolha dos artigos, foram selecionados os publicados entre 2011 a 2021 em periódicos nacionais e internacionais. Como critérios de inclusão para a realização deste estudo, foram considerados artigos científicos que abordavam o uso incorreto dos opioides, bem como os malefícios dessa prática, além de revisões de literaturas que descrevem os efeitos farmacológicos e toxicológicos dessas drogas no organismo devido à automedicação, uso crônico dos mesmos ou por erros de prescrições. Foram excluídos estudos que tinha publicação fora do período e idiomas mencionados acima, ou com conteúdos que não tinham relação com o tema proposto. Por fim, foram selecionados 34 trabalhos.

\section{Discussão}

\section{Principais opioides utilizados indiscriminadamente}

Considerando que os estímulos dolorosos levam a um grande desconforto, é necessária intervenção que se dá por meio de tratamento com opções não farmacológicas e farmacológicas. Quando ocorre a utilização de fármacos a classe medicamentosa utilizada é a dos analgésicos. Para um tratamento preciso e uma melhor resposta farmacológica é importante seguir alguns critérios como: respeitar o intervalo e dose terapêutica dos medicamentos, a resposta prévia do paciente à medicação e o controle dos efeitos colaterais (Hennemann-Krause, 2012).

A prescrição de medicamentos de uma forma geral deve ter um monitoramento contínuo e ser rigorosa e bastante criteriosa. Sabe-se que para o alívio da dor e inflamação são utilizados os anti-inflamatórios não esteroidais (AINES), contudo os opioides são os prescritos para manejo de dores moderadas e intensas (Ely et al., 2015). Em relação a sua utilização, os opioides são indicados para controle da dor neuropática ou mistas, lombalgias, cefaleias, artrite, condições pré e pós-operatórias, anestesias, além do uso no tratamento da dor crônica em pacientes oncológicos (Lima \& Pereira, 2017). Normalmente, no tratamento com opioide, recomenda-se que a princípio sejam utilizados os mais simples e menos invasivos (Magalhães et al., 2019). Tal explicação pode ser observada na Figura 1.

Os opioides considerados fracos são indicados para controle da dor quando analgésicos comuns são insuficientes, alguns AINEs podem ser utilizados associados com Codeína e Tramadol. Quando esses não são suficientes são indicados medicamentos da mesma classe com ação mais potente como: morfina, metadona, oxicodona, fentanil (Tabela 1). O limite da dose nesses casos é o aparecimento de efeitos adversos intoleráveis e excessivos (Hennemann-Krause, 2012). Existem controvérsias quanto à utilização dos 
opioides, e alguns dados são importantes para uma melhor compreensão, pois opioides com dose eficaz para um paciente podem ser perigosos para outros utilizando a mesma dosagem, por isso é muito importante que as prescrições sejam avaliadas e que os pacientes recebam orientação durante o acompanhamento (Magalhães et al., 2019).

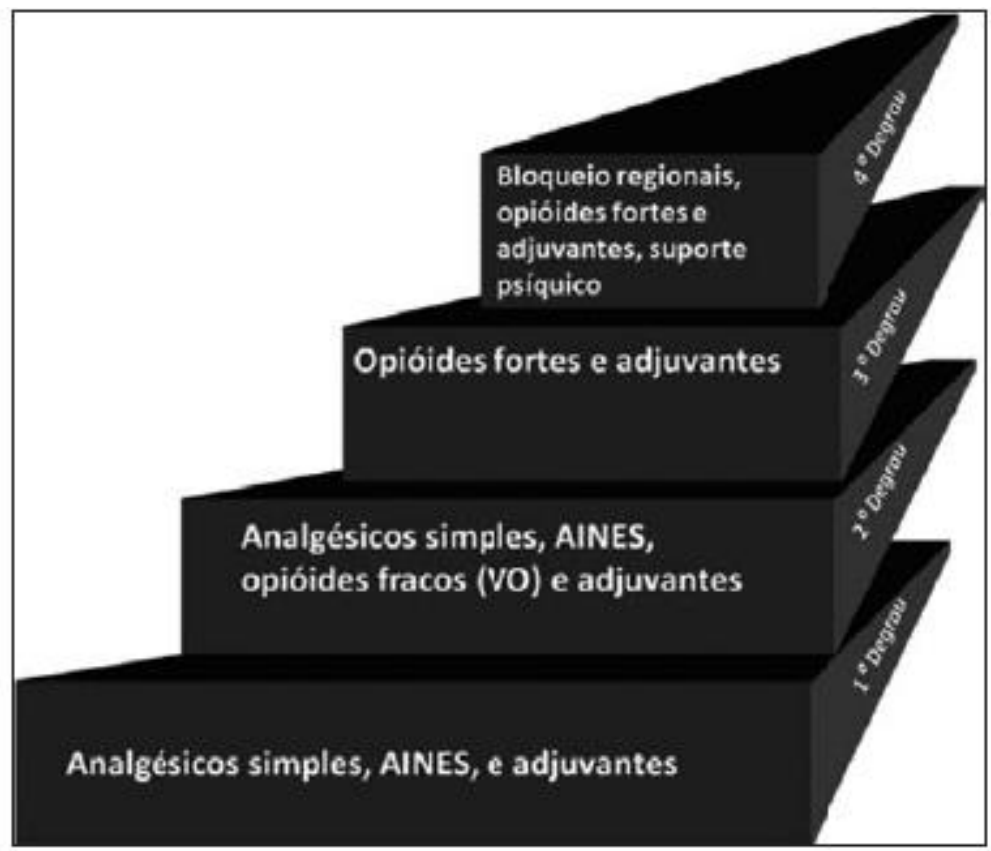

Figura 1. Escala modificada da dor em quatro degraus. Fonte: Kraychete et al. (2013).

Tabela 1. Analgésicos opioides.

\begin{tabular}{|c|c|c|c|}
\hline Fármacos & Apresentação, doses & $\begin{array}{c}\text { Doses } \\
\text { terapêuticas/intervalo }\end{array}$ & Efeito (início, pico/fim) \\
\hline Fentanil & Adesivos, 5,10 e $20 \mathrm{mg}$ & $5-20 \mathrm{mg} / 7$ dias & $24 \mathrm{~h} / 72 \mathrm{~h}$ \\
\hline Morfina & $\begin{array}{c}\text { Cápsulas, } 10 \text { e } 30 \mathrm{mg} \\
\text { Solução oral, } 10 \mathrm{mg} / \mathrm{mL} \\
\text { Ampolas, } 1 \mathrm{~mL}-10 \mathrm{mg} / \mathrm{mL} \\
\end{array}$ & $5-200 \mathrm{mg} / 4 \mathrm{~h}$ (dose oral) & $15 \mathrm{~min} / 2 \mathrm{~h} / 4 \mathrm{~h}$ \\
\hline Morfina LC & Cápsulas, 30, 60 e $100 \mathrm{mg}$ & $30-100 \mathrm{mg} / 8 \mathrm{~h}$ a $12 \mathrm{~h}$ & $1 \mathrm{~h} / 6 \mathrm{~h} / 14 \mathrm{~h}$ \\
\hline Oxicodona & Cápsulas, 10, 20 e $40 \mathrm{mg}$ & $10-40 \mathrm{mg} / 12 \mathrm{~h}$ & $1 \mathrm{~h} / 8 \mathrm{~h} / 25 \mathrm{~h}$ \\
\hline Hidromorfona & $\begin{array}{l}\text { Comprimido de liberação prolongada, } \\
8 \mathrm{mg}, 16 \mathrm{mg}, 32 \mathrm{mg} \\
\end{array}$ & $8-32 \mathrm{mg} / 24 \mathrm{~h}$ & $6 \mathrm{~h}$ a $8 \mathrm{~h} / 24 \mathrm{~h}$ \\
\hline Metadona & $\begin{array}{l}\text { Cápsulas, } 5 \text { e } 10 \mathrm{mg} \\
\text { Ampolas, } 10 \mathrm{mg} / \mathrm{mL} \\
\end{array}$ & $10-50 \mathrm{mg} / 6-12 \mathrm{~h}$ & $1 \mathrm{~h} / 12 \mathrm{~h} / 25 \mathrm{~h}$ \\
\hline Brupenorfina & Adesivos, 5,10 e $20 \mathrm{mg}$ & $5-20 \mathrm{mg} / 7 \mathrm{dias}$ & $18 \mathrm{~h}$ a $24 \mathrm{~h} / 72 \mathrm{~h} / 7$ dias \\
\hline
\end{tabular}

Fonte: Silva et al. (2020b).

Prescrever opioides de forma consciente e racional, ainda é um desafio para os profissionais de saúde capacitados, onde é essencial reconhecer as necessidades de cada paciente para que a terapêutica seja eficaz e sem malefícios, porque mesmo em ambientes hospitalares são observadas prescrições de formas pouco racional (Ribeiro \& Costa, 2015). Diante do exposto, para o uso seguro dos opioides são necessários cuidados com a titulação que é um método empregado para determinação da dose ideal de uma substância, ou seja, as doses vão sendo aumentadas de forma gradativa, até a obtenção de uma analgesia efetiva, com efeitos adversos toleráveis. Essa prática tem como objetivo uma dose que traga a melhora da dor e/ou reduza a intensidade (Kraychete et al., 2013). Além de ajustes individuais e sua redução gradual (Coluzzi et al., 2016; Melo et al., 2020b). A tabela 2 apresenta os critérios para uma indicação consciente dos opioides.

Segundo dados epidemiológicos coletados, a prevalência anual do uso de opioides na Europa, no ano de 2017, foi de 0,7\% da população com idade entre 15-54 anos, não sendo mencionado outras características. Nesse mesmo ano nos Estados Unidos da América (EUA), a prevalência foi de 4\%, nas idades entre 12-17 anos e acima de 26 anos; e entre 15-25 anos foi de 8\% (Merz, 2019; Servin et al., 2020).

Segundo Krawczyk et al. (2018) e Servin et al. (2020) as farmácias no Brasil registraram um aumento de 1.601.043, prescrições em 2009 e 9.045.945, em 2015. As prescrições mais prevalentes foram para o uso 
ambulatorial, destacando os produtos de codeína, representando 98,9\% e 98,1\% das prescrições de opioides investigadas em 2009 e 2015, respectivamente. No ano de 2009, oxicodona e fentanil foram os responsáveis por $0,85 \%$ e $0,19 \%$ das prescrições de opioides.

Tabela 2. Critérios a serem observados antes da prescrição de opioides.

\begin{tabular}{c}
\hline Indicação segura - avaliação \\
\hline 1) Avaliação da dor \\
2) Quadro clínico geral \\
3) História psicossocial \\
4) Condições psiquiátricas \\
5) Eventual abuso de substância ilícita \\
6) Termo de consentimento informado para o uso de opioide (portaria da Agência Nacional \\
de Vigilância Sanitária (ANVISA) \\
1) Avaliação da dor
\end{tabular}

Fonte: Adaptado de Kraychete et al. (2013).

O uso deve ser consciente, pois, esses efeitos colaterais podem ser irreversíveis como num estudo feito em 2009, aproximadamente 30\% das mortes relacionadas a analgésicos nos Estados Unidos foram atribuídas à metadona, embora essa droga representasse apenas $2 \%$ do consumo de opioides. Não há dúvida de que os analgésicos opioides expõem os usuários ao risco de intoxicação e até de morte. Portanto, o uso de metadona e dos demais fármacos dessa classe deve incluir avaliação sistemática e medidas para reduzir esse risco, como educação do paciente e início do tratamento ou monitoramento dos sintomas durante a titulação da dose (Barbosa Neto et al., 2015).

Portanto, são imprescindíveis mais pesquisas sobre dor e o distúrbio do uso abusivo dessas drogas, reforço constante na educação de prescritores, reconhecimento da dependência em tempo ágil, além, claro, da garantia de acesso aos tratamentos eficazes contra a adição desses medicamentos sem necessidade crônica (Leal, 2020).

\section{Efeitos toxicológicos decorrentes da utilização}

A analgesia a nível central se dá através da interação com os receptores opioides inibindo diretamente as etapas da nocicepção e com isso a transmissão de informações de nociceptores que se iniciam a partir do corno dorsal da medula espinhal e tem a capacidade de ativar as vias descendentes inibitórias da dor. Os agonistas do receptor opioide, que são os ligantes que ativam esses receptores, ativam a proteína Gi, levando à diminuição do AMP-cíclico (AMPc) nos neurônios e com isso há o fechamento dos canais de cálcio voltagem dependentes nas terminações pré-sinápticas, impedindo a liberação dos neurotransmissores na fenda sináptica. Ocorre também à abertura dos canais de potássio, levando um influxo dos íons de $\mathrm{K}+$ dentro do neurônio, e esses íons levam a hiperpolarização do neurônio pós-sináptico, tendo como resultado uma redução da excitabilidade neuronal, bloqueando a transmissão do estímulo doloroso (Martins et al., 2012; Martinez et al., 2014; Bezerra et al., 2018). Sendo assim, são drogas bastante administradas por sua potente e rápida ação, contudo os efeitos adversos dos opioides também são comuns, entre eles: dependência, depressão respiratória, constipação intestinal, prurido, sonolência e náuseas/vômitos, apenas um número pequeno apresentam parada respiratória (Posso et al., 2013).

Além dos efeitos citados, uso abusivo de opioides pode produzir um quadro de intoxicação, caracterizado por sedação, euforia e miose (exceto meperidina, que causa midríase). A intoxicação de forma acidental ocorre em indivíduos com baixa tolerância à substância, em associação a outras drogas ou a uma variação abrupta da dose, incluindo predisposições genéticas (Campos et al., 2020).

Um outro efeito toxicológico sério, é a dependência, e ela ocorre devido a combinação de fatores que incluem a predisposição genética, perfil psicológico, contexto sociocultural e exposição ao fármaco (Nascimento \& Sakata, 2011). Pessoas que tem entre 18-24 anos, sexo masculino, queixa de dor em diversas partes do corpo, além de histórico anterior de abuso de álcool, drogas ilícitas, tabagismo, transtornos psiquiátricos, uso de Cannabis, uso de psicotrópicos, aumento do grau de tolerância à dor são os mais propensos a apresentarem dependência (Leal, 2020). A abstinência é um outro malefício e essa síndrome está relacionada à interrupção repentina de opioides após um período prolongado de uso. Os opioides de curta ação, como a heroína, geralmente provocam sinais e sintomas de abstinência dentro de 8-12 horas após a última dose. Se não for tratada, atinge o pico em 36-72 horas e geralmente diminui substancialmente 
em 5 dias. Em opioides de ação prolongada como a metadona, à abstinência pode atingir um pico entre 5-6 dias, e a síndrome geralmente não cessa por 14-21 dias (Fareed et al., 2011).

Em alguns casos de intoxicação, síndrome de abstinência e até mesmo em situações em que os pacientes ficam agressivos devido a alterações comportamentais que essas drogas induzem, é preciso uma intervenção farmacológica e são utilizados compostos agonistas que se ligam competitivamente aos mesmos receptores que atuam no efeito dessas drogas, e com isso evitam ou obstruem esses efeitos adversos. Sendo assim, a terapia farmacológica inclui o uso de agonistas (buprenorfina ou metadona) ou de um antagonista (naloxona e naltrexona) (Webster \& Fine, 2012; Melo et al., 2020b).

As ações farmacodinâmicas dos opioides agonistas geralmente são semelhantes, e envolvem analgesia, sedação, inconsciência, sonolência, etc. Já os antagonistas como a naloxona, podem reverter à ação dos agonistas, assim como a de alguns agonistas/antagonistas e agonistas parciais, como a buprenorfina (Gozzani, 2020). O quadro 1 apresenta informações sobre o uso desses fármacos na terapia de efeitos adversos.

Quadro 1. Terapia farmacológica para retirada de opioides.

\begin{tabular}{|c|c|c|c|c|}
\hline Fármaco & Via de administração & Risco de abuso & Posologia & Benefícios \\
\hline Buprenorfina & Sublingual ou parenteral & $\begin{array}{l}\text { Potencialmente } \\
\text { baixo }\end{array}$ & $8-16 \mathrm{mg} / \mathrm{dia}$ & $\begin{array}{l}\text { É eficaz, ela suprime os sintomas } \\
\text { de abstinência e é bem tolerada e } \\
\text { segura }\end{array}$ \\
\hline Metadona & Oral & $\begin{array}{l}\text { Perfil risco de } \\
\text { segurança alto }\end{array}$ & 80 a 120 mg por dia & $\begin{array}{l}\text { Ação prolongada, tem potencial } \\
\text { de aliviar o desejo narcótico, } \\
\text { suprimir a síndrome de } \\
\text { abstinência e bloquear os efeitos } \\
\text { eufóricos associados à heroína }\end{array}$ \\
\hline Naloxona & $\begin{array}{l}\text { O uso oral é limitado, } \\
\text { endovenoso. }\end{array}$ & $\begin{array}{l}\text { O uso com } \\
\text { Buprenorfina } \\
\text { pode precipitar es } \\
\text { tados de abstinên } \\
\text { cia consideravelm } \\
\quad \text { ente grave. }\end{array}$ & $0,4 \mathrm{mg}$ & $\begin{array}{l}\text { Utilizado na depressão respiratória } \\
\text { induzida por opioides, mas tem } \\
\text { uma meia vida curta, precipita a } \\
\text { retirada em pacientes dependentes } \\
\text { e, portanto, a reversão } \\
\text { persistente de opioides de ação } \\
\text { prolongada } \\
\text { deve ser administrada por doses } \\
\text { e infusões tituladas. }\end{array}$ \\
\hline Naltrexona & Oral & & $\begin{array}{c}\text { Três vezes por semana, } \\
\text { em dose de } 50 \text { a } 100 \mathrm{mg}, \\
\text { em dias de semana e de } \\
100 \text { a } 150 \text { mg nos fins de } \\
\text { semana }\end{array}$ & $\begin{array}{c}\text { É utilizado em paciente em } \\
\text { abstinência é relativamente seguro } \\
\text { para o tratamento de longo prazo. }\end{array}$ \\
\hline
\end{tabular}

Fonte: Adaptado de Melo et al. (2020b).

\section{Importância da atenção farmacêutica no uso racional de medicamentos desta classe}

Salientando os efeitos adversos, à medida que a população para a qual os opioides são indicados cresceu, devido ao número de prescrições de opioides, também aumentaram os indicadores de uso indevido, abuso, morbidade e mortalidade relacionados aos opioides (Kanouse \& Compton, 2015). A intervenção do farmacêutico é muito importante, pois é um profissional que possui habilidades de conhecimentos sobre medicamentos, e poderá orientar tanto o paciente quanto o familiar ou acompanhante em relação ao uso racional dos medicamentos, facilitando a compreensão desde a sua prescrição até as orientações quanto ao uso e possíveis interações medicamentosas, esses trabalhadores devem estar capacitados em termos de conhecimentos, habilidades e atitudes para elaborar e atuar em ações específicas diante das necessidades apresentadas, de maneira integrada com as demais práticas da rede de cuidado social (Costa et al., 2019). 
Os farmacêuticos podem contribuir para prescrições adequadas de opioides, entendendo também das necessidades do acesso aos opioides para os pacientes que precisam desses medicamentos, esse auxílio junto à equipe médica melhora a terapia. As organizações médicas e os conselhos devem continuar apoiando a ações educativas para os profissionais, e divulgando informações seguras para os prescritores, minimizando os riscos de uso indiscriminado (Hadland et al., 2019).

Algumas estratégias podem ser adotadas, visando solucionar ou amenizar tais problemas apresentados, como: utilização de escalas de dor para facilitar a seleção dos medicamentos e monitoramento da dor do paciente; avaliação multidimensional; escolha de opioides de acordo com cada particularidade apresentada, ou seja, um tratamento mais individualizado, observando cada fisiopatologia apresentada; encaminhamento para demais especialidades quando necessário, ocorrendo uma interação entre os diversos profissionais/especialidades e pacientes; educação de pacientes, familiares e da equipe para uma otimização do tratamento; fornecimento de apoio psicossocial; estabelecer expectativas realistas e objetivas em relação à dor. Além da educação em saúde para um melhor gerenciamento da dor por parte dos profissionais da área da saúde e aumentar a disponibilidade de opioides (Silva et al., 2020b).

Aconselha-se que as instituições de saúde implementem políticas mais detalhadas para pacientes que fazem uso de analgésicos opioides, facilitando os procedimentos e monitoramento clínico dos mesmos. Espera-se que este estudo possa ampliar os estudos sobre essa temática de bastante relevância social, incentivando a produção científica com novos estudos nessa área. Elaborando fontes seguras sobre o uso dos opioides, gerando mais foco e discussão em toda equipe multidisciplinar (Henrique et al., 2017).

\section{Considerações finais}

O tratamento de processos dolorosos é um desafio dos profissionais de saúde, e ter uma terapia eficaz sem grandes prejuízos ainda é um obstáculo quando se trata de opioides. Porque o uso inadequado traz danos físicos como depressão respiratória, constipação, e outros que junto com os sintomas físicos afetam o psicológico das pessoas como abstinência e dependência. Dessa forma, fica clara a importância do trabalho multiprofissional, pois, somente com uma integração da equipe, levantando discussões sobre o uso seguro dos opioides haverá avanço nesse contexto. É fundamental criar ações educativas para a população trazendo informações atualizadas, claras e de fácil entendimento, além da orientação e acompanhamento individual caso a caso.

\section{Referências}

Barbosa Neto, J. O., Garcia, M. A., \& Garcia, J. B. S. 2015. Revisitando a metadona: farmacocinética, farmacodinâmica e uso clínico. Revista Dor, 16(1), 60-66.

Bezerra, K. G. D., Barreto, F. D. S., Lopes, J. C. F., Leite, J. M. S., \& Oliveira, F. S. 2017. Hiperalgesia induzida por opioides: uma revisão. II Conbracis - Congresso Brasileiro de Ciências da Saúde, 1-11.

Bicca, C., Ramos, F. L. P., Campos, V. R., Assis, F. D., Pulchinelli Júnior, A., Lermnen Júnior, N., Marques, A. C. P. R., Ribeiro, M., Laranjeira, R. R., \& Andrada, N. C. 2012. Projeto Diretrizes: Abuso e Dependência dos Opioides e Opiáceos, 1-30.

Campos, H. S. P., Oliveira, J. N., Arcanjo, N. A., Oliveira, N. M. G., Rosa, T. C. T. O., \& Reis, L. F. A. 2020. Opioides: toxicidade e efeitos indesejados. Única Cadernos Acadêmicos, 3(1), 1-12.

Coluzzi, F., Taylor, R., Pergolizzi, J. V., Mattia, C., \& Raffa, R. B. 2016. Orientação para boa prática clínica para opioides no tratamento da dor: os três" Ts"-titulação (teste), ajustes (individualização), transição (redução gradual). Revista Brasileira de Anestesiologia, 66, 310-317.

Costa, S. T., Alves, F. C. E. F., \& de Jesus, M. R. (2019). Auxiliar farmacêutico na saúde do idoso quanto ao uso do medicamento controlado. Revista GeTeC, 8(21).

Ely, L. S., Engroff, P., Guiselli, S. R., Cardoso, G. C., Morrone, F. B., \& Carli, G. A. D. 2015. Uso de antiinflamatórios e analgésicos por uma população de idosos atendida na Estratégia Saúde da Família. Revista Brasileira de Geriatria e Gerontologia, 18(3), 475-485.

Fareed, A., Stout, S., Casarella, J., Vayalapalli, S., Cox, J., \& Drexler, K. 2011. Illicit opioid intoxication: diagnosis and treatment. Substance Abuse: Research and Treatment, 5, 17-25.

Gozzani, J. L. 2020. Opióides e antagonistas. Brazilian Journal of Anesthesiology, 44(1), 65-73. 
Hadland, S. E., Rivera-Aguirre, A., Marshall, B. D., \& Cerdá, M. 2019. Association of pharmaceutical industry marketing of opioid products with mortality from opioid-related overdoses. JAMA Network Open, 2(1), e186007.

Hennemann-Krause, L. 2012. Aspectos práticos da prescrição de analgésicos na dor do câncer. Revista Hospital Universitário Pedro Ernesto, 11(2), 38-49.

Henrique, D. D. M., Silva, L. D. D., Camerini, F. G., Andrade, K. B. S., Pereira, S. R. M., \& Fassarella, C. S. 2017. Aprazamento seguro da terapia analgésica com opioides no paciente queimado: Um estudo transversal. Revista Enfermagem UERJ, 25, e28082.

Kanouse, A. B., \& Compton, P. 2015. The epidemic of prescription opioid abuse, the subsequent rising prevalence of heroin use, and the federal response. Journal of Pain \& Palliative Care Pharmacotherapy, 29(2), 102-114.

Krawczyk, N., Greene, M. C., Zorzanelli, R., \& Bastos, F. I. 2018. Rising trends of prescription opioid sales in contemporary Brazil, 2009-2015. American Journal of Public Health, 108(5), 666-668.

Kraychete, D. C., Siqueira, J. T. T. D., \& Garcia, J. B. S. 2013. Recomendações para uso de opioides no Brasil: parte I. Revista Dor, 14(4), 295-300.

Kraychete, D. C., Siqueira, J. T. T. D., \& Garcia, J. B. S. 2014. Recomendações para uso de opioides no Brasil: Parte II. Uso em crianças e idosos. Revista Dor, 15(1), 65-69.

Lima, M. B., \& Pereira, M. C. A. 2017. Constipação intestinal em pacientes tratados com opioides: uma revisão integrativa. Revista Brasileira em Promoção da Saúde, 30(2), 275-282.

Melo, H. M. A., Lima, J. T. O., Souto, F. O., \& Lima Filho, J. L. 2020a. Prescrição de opioides em idosos com câncer: evidências sobre imunossupressão e seu impacto na prática clínica. Revista Eletrônica Acervo Saúde, 12(5), e2868-e2868.

Melo, A. P., Fujii, Y. W. H., Rangel, M. P., \& Nishida, F. S. 2020b. Retirada de opioides: uma revisão bibliográfica. Brazilian Journal of Development, 6(9), 67098-67112.

Leal, R. 2020. Uso indevido e dependência de opioides: da prevenção ao tratamento. Revista de Medicina de Família e Saúde Mental, 2(1), 29-44.

Magalhães, A. R., Souza, M. M. S., de Lima, J. P., \& de Oliveira, I. C. M. 2019. Manejo clínico na utilização de opioides no controle da dor no paciente oncológico. Encontro de Extensão, Docência e Iniciação Científica (EEDIC). Centro Universitário Católica de Quixadá, 1-6.

Martinez, J. E., Pereira, G. A. F., Ribeiro, L. G. M., Nunes, R., Ilias, D., \& Navarro, L. G. M. 2014. Estudo da automedicação para dor musculoesquelética entre estudantes dos cursos de enfermagem e medicina da Pontifícia Universidade Católica-São Paulo. Revista Brasileira de Reumatologia, 54(2), 90-94.

Martins, R. T., Almeida, D. B. D., Monteiro, F. M. D. R., Kowacs, P. A., \& Ramina, R. 2012. Receptores opioides até o contexto atual. Revista Dor, 13(1), 75-79.

Minson, F. P., Garcia, J. B. S., de Oliveira Júnior, J. O., \& de Siqueira, J. T. T. 2012. Opioides-Farmacologia básica. In: Carvalho, R. T., \& Parsons, H. A. Manual de Cuidados Paliativos, ANCP. $2^{\text {a }}$ ed. 123p.

Nascimento, D. C. H., \& Sakata, R. K. 2011. Dependência de opioide em pacientes com dor crônica. Revista Dor, 12(2), 160-165.

Neves, J. R. D. S. 2016. Análise toxicológica de opióides em contexto forense. Dissertação (Mestrado) Universidade Fernando Pessoa, Faculdade Ciências da Saúde, Porto, 88p.

Posso, M. B. S., Giaretta, V. M. D. A., Santanna, A. L. G. D., Ranzani, R. C. M., \& Gouvea, Á. L. 2013. Percepção dos enfermeiros sobre o tratamento da dor crônica não maligna com opioides. Revista Dor, 14(1), 7-11.

Ribeiro, H. D. S. S., \& Costa, J. M. 2015. Acompanhamento farmacoterapêutico de idosos em uso de analgésicos opioides em um hospital de ensino. Revista Brasileira de Farmácia Hospitalar e Serviços de Saúde, 6(1), 18-23.

Servin, E. T. N., Filipe, L. N. S. M., da Cunha Leal, P., de Oliveira, C. M. B., Moura, E. C. R., \& de Sousa Gomes, L. M. R. 2020. A crise mundial de uso de opióides em dor crônica não oncológica: causas e estratégias de manejo e relação com o Brasil. Brazilian Journal of Health Review, 3(6), 18692-18712.

Silva, J. C. S., Souza, F. C. R., Aoyama, E. A. 2020. A incidência do uso indiscriminado de medicamentos. Revista Brasileira Interdisciplinar de Saúde, 2(1), 95-99. 
Silva, L. J. D., Mendanha, D. M., \& Gomes, P. P. 2020b. O uso de opioides no tratamento da dor oncológica em idosos. Brazilian Journal of Pain, 3(1), 63-72.

Merz, F. 2018. United Nations Office on Drugs and Crime: World Drug Report 2017. 2017. SIRIUS-Zeitschrift für Strategische Analysen, 2(1), 85-86.

Webster, L. R., \& Fine, P. G. 2012. Review and critique of opioid rotation practices and associated risks of toxicity. Pain Medicine, 13(4), 562-570.

Wiffen, P. J., Wee, B., Derry, S., Bell, R. F., \& Moore, R. A. 2017. Opioids for cancer pain-an overview of Cochrane reviews. Cochrane Database of Systematic Reviews, 7(7), CD012592.

\section{Minicurrículo}

Lorena Santos Sousa. Graduanda no curso de Farmácia da Universidade Salvador, Salvador, Bahia (BA), Brasil.

Milena Silva Cerqueira Pinheiro. Graduanda no curso de Farmácia da Universidade Salvador, Salvador, Bahia (BA), Brasil.

Juliana Lima Gomes Rodrigues. Graduada em Farmácia pela Universidade Federal da Bahia, Mestre em Farmácia pela Universidade Federal da Bahia, Farmacêutica servidora pública do Instituto de Ciências da Saúde (ICS/UFBA), Docente da Universidade Salvador (UNIFACS).

Como citar: Sousa, L.S., Pinheiro, M.S.C., \& Rodrigues, J.L.G. 2021. Uso indiscriminado dos opioides e suas consequências. Pubsaúde, 6, a190. DOI: https://dx.doi.org/10.31533/pubsaude6.a190

Recebido: 17 mai. 2021.

Revisado e aceito: 4 jun. 2021.

Conflito de interesse: os autores declaram, em relação aos produtos e companhias descritos nesse artigo, não ter interesses associativos, comerciais, de propriedade ou financeiros que representem conflito de interesse.

Licenciamento: Este artigo é publicado na modalidade Acesso Aberto sob a licença Creative Commons Atribuição 4.0 (CC-BY 4.0). 